\title{
Asymptotic safety with Majorana fermions and new large $N$ equivalences
}

\author{
Andrew D. Bond, Daniel F. Litim $\odot$, and Tom Steudtner \\ Department of Physics and Astronomy, University of Sussex, Brighton BN1 9QH, United Kingdom
}

(Received 6 December 2019; accepted 15 January 2020; published 10 February 2020)

\begin{abstract}
Using Majorana fermions and elementary mesons we find new massless quantum field theories with weakly interacting ultraviolet fixed points. We also find new classes of large $N$ equivalences among $S U$, $S O$, and $S p$ gauge theories with different types of matter fields and Yukawa interactions. Results include a triality of asymptotically safe theories and dualities between asymptotically free matter-gauge theories with identical fixed points, phase diagrams, and scaling exponents. Implications for conformal field theory and orbifold reductions are indicated.
\end{abstract}

DOI: 10.1103/PhysRevD.101.045006

\section{INTRODUCTION}

Equivalences or dualities between seemingly different theories can provide valuable insights into the dynamics of quantum fields at weak and strong coupling. Well-known examples include equivalences between $S U, S O$, and $S p$ gauge theories in the limit where the rank of the gauge group is large [1], electric-magnetic duality in supersymmetric theories [2], the seminal AdS/CFT conjecture [3], or equivalences between theories related by orbifold/ orientifold projections [4-9] where parent and child theories achieve coinciding perturbative expansions in the planar limit, and, under some conditions, nonperturbative equivalence [10,11]. Large $N$ equivalences have also seen many applications in QCD-like theories including on the lattice [12-14].

On a different tack, the discovery of interacting ultraviolet fixed points in QCD-like theories, first conjectured in [15], has sparked a lot of interest [16-30]. It has led to a general classification of four-dimensional (4D) quantum field theories including necessary and sufficient conditions and strict no-go theorems for weakly interacting fixed points [18,27]. In the large $N$ limit, proofs for asymptotic safety with Dirac fermions are available with [25] and without supersymmetry [16,22-24]. Key ingredients are Yukawa interactions, which can stabilize nonfree gauge couplings [18]. At finite $N$, these ideas are used to UV complete the standard model $[20,26,28]$ and to study aspects of flavor and vacuum stability $[29,30]$.

In this paper, we explain how asymptotic safety materializes in theories with Majorana fermions and elementary

Published by the American Physical Society under the terms of the Creative Commons Attribution 4.0 International license. Further distribution of this work must maintain attribution to the author(s) and the published article's title, journal citation, and DOI. Funded by SCOAP ${ }^{3}$. mesons, and how this compares to settings with Dirac fermions. With the help of perturbation theory, the renormalization group (RG), negative dimensionality theorems, and ideas from string theory, we also put forward new classes of large $N$ equivalences among gauge-Yukawa theories with different gauge or global symmetries, and different types of matter fields. Results include a triality of asymptotically safe theories with $S U, S O$, or $S p$ gauge groups with identical phase diagrams and scaling exponents at ultraviolet critical points, and dualities between asymptotically free gauge-matter theories with identical infrared critical points and more.

\section{MAJORANA FERMIONS}

We consider non-Abelian gauge theories coupled to Majorana fermions $\Psi_{i}$ and singlet complex scalar fields $H_{i j}$. Majorana fermions are their own charge conjugates $\Psi^{c}=\Psi$ whose left- and right-handed chiral components

$$
\Psi=\frac{1}{\sqrt{2}}\left(\psi, \psi^{c}\right)^{\top}
$$

relate to the same Weyl field $\psi$ with charge conjugation $\psi^{c}=\varepsilon \psi^{*}$ and $\varepsilon=\left(\begin{array}{cc}0 & 1 \\ -1 & 0\end{array}\right)$. Real representations ensure that both Weyl components undergo identical gauge transformations, whose generators are purely imaginary and antisymmetric $t^{a}=-\left(t^{a}\right)^{\top}=-\left(t^{a}\right)^{*}$. For theories with chiral Yukawa interactions the requirement for real representations can be weakened to include pseudoreal ones that are real up to a transformation $\left(t^{a}\right)^{\top}=-M t^{a} M^{-1}$. In either case chiral gauge anomalies cancel due to the vanishing of

$$
d^{a b c} \equiv \frac{1}{2} \operatorname{Tr}\left[t^{a}\left\{t^{b}, t^{c}\right\}\right]=0 .
$$

To ensure strict perturbative control, we use a suitable large $N$ limit [31] that necessitates the Majorana fermions to be 
TABLE I. Dimensions and quadratic Casimirs of fundamental and adjoint representations with Dynkin index $S_{2}^{R}=\frac{1}{2}$.

\begin{tabular}{lccc}
\hline \hline Invariant & $S U(N)$ & $S O(N)$ & $S p(N)$ \\
\hline$d_{R}$ & $N$ & $N$ & $N$ \\
$C_{2}^{R}$ & $\frac{1}{2}(N-1 / N)$ & $\frac{1}{4}(N-1)$ & $\frac{1}{4}(N+1)$ \\
$d_{G}$ & $N^{2}-1$ & $\frac{1}{2} N(N-1)$ & $\frac{1}{2} N(N+1)$ \\
$C_{2}^{G}$ & $N$ & $\frac{1}{2}(N-2)$ & $\frac{1}{2}(N+2)$ \\
\hline \hline
\end{tabular}

in the fundamental representation. The latter implies that unitary or any of the exceptional gauge groups are excluded, which leaves us with orthogonal or symplectic gauge groups.

Orthogonal gauge symmetry $S O(N)$.-We begin with a theory of $N_{f}$ Majorana fermions in the fundamental representation of an $S O(N)$ gauge theory, interacting with gaugesinglet complex scalar fields $H$. The theory has a global $S U\left(N_{f}\right)$ flavor symmetry with the Weyl components transforming in the fundamental and the scalars in the two-index symmetric representation $H_{i j}=H_{(i j)}$. The Lagrangian reads

$$
\begin{aligned}
L= & -\frac{1}{4} F_{\mu \nu}^{a} F^{a \mu \nu} \\
& +\operatorname{Tr}\left(\psi^{\dagger} i \sigma^{\mu} D_{\mu} \psi\right)+\operatorname{Tr}\left(\partial_{\mu} H^{\dagger} \partial^{\mu} H\right) \\
& -\frac{1}{2} y \operatorname{Tr}\left(\psi^{\top} h H \varepsilon \psi+\psi^{\dagger} h H^{\dagger} \varepsilon \psi^{*}\right) \\
& -u \operatorname{Tr}\left(H^{\dagger} H\right)^{2}-v\left(\operatorname{Tr} H^{\dagger} H\right)^{2},
\end{aligned}
$$

where $F_{\mu \nu}^{a}$ denotes the non-Abelian field strength, the trace sums over gauge and flavor indices, and gauge contractions of fermion bilinears $(\chi \xi)=\chi_{\alpha} h^{\alpha \beta} \xi_{\beta}$ are symmetric with $h^{\alpha \beta}=h^{\beta \alpha}$ and $h^{\alpha \beta} h_{\beta \gamma}=\delta^{\alpha}{ }_{\gamma}$. The four canonically marginal couplings $\{g, y, u, v\}$ of the perturbatively renormalizable theory are the gauge, Yukawa, single, and double trace quartic, respectively.

Next, we investigate the renormalization group equations for the running couplings [32-35] and search for perturbative fixed points of the theory [18]. Perturbative control is achieved using a Veneziano limit [31] where the dimension of the fundamental representation $d_{R}$ and the number of fermion flavors $N_{f}$ are sent to infinity while their ratio is kept fixed (see Table I for our conventions of grouptheoretical parameters). The parameter

$$
\epsilon=\frac{N_{f}}{N}-\frac{11}{2}
$$

becomes continuous and may take any value within the range $-\frac{11}{2}<\epsilon<\infty$. For $\epsilon<0$, the theory is asymptotically free, while asymptotic freedom is absent for $\epsilon>0$. Following 't Hooft [36], we introduce rescaled couplings suitable for a planar or large $N$ limit,

$$
\alpha_{x}=\frac{d_{R} x^{2}}{(4 \pi)^{2}}, \quad \alpha_{u}=\frac{N_{f} u}{(4 \pi)^{2}}, \quad \alpha_{v}=\frac{N_{f}^{2} v}{(4 \pi)^{2}},
$$

where $x=g$ or $y$, and beta functions $\beta_{i} \equiv d \alpha_{i} / d \ln \mu$. To the leading nontrivial orders in perturbation theory that is two loop in the gauge and one loop in the Yukawa and quartic beta functions, we find

$\beta_{g}=\alpha_{g}^{2}\left[\frac{2}{3} \epsilon+\left(\frac{25}{4}+\frac{13}{6} \epsilon\right) \alpha_{g}-\frac{1}{2}\left(\frac{11}{2}+\epsilon\right)^{2} \alpha_{y}\right]$,

$\beta_{y}=\alpha_{y}\left[\left(\frac{13}{2}+\epsilon\right) \alpha_{y}-3 \alpha_{g}\right]$,

$\beta_{u}=4 \alpha_{u}^{2}+2 \alpha_{y} \alpha_{u}-\left(\frac{11}{2}+\epsilon\right) \alpha_{y}^{2}$,

$\beta_{v}=2 \alpha_{v}^{2}+8 \alpha_{u} \alpha_{v}+6 \alpha_{u}^{2}+2 \alpha_{y} \alpha_{v}$.

In any $4 \mathrm{D}$ quantum field theory, the weakly coupled fixed point solutions to $\beta_{i}=0$ are either of the Banks-Zaks or of the gauge-Yukawa type $[18,27]$. For small $\epsilon$ they arise as a strict power series in $\epsilon$ where subleading terms up to order $\epsilon^{n}$ are obtained from the loop order $(n+1, n, n)$ in the gauge, Yukawa, and quartic beta functions $[23,24]$. Also, any weakly coupled fixed point corresponds to a (unitary) conformal field theory [37].

Our results are summarized in Table II. In the regime with asymptotic freedom the theory (3) with (6) displays a Banks-Zaks fixed point $\mathrm{FP}_{1}$. Infrared gauge-Yukawa fixed points are absent. In the regime where asymptotic freedom is lost, the gauge-Yukawa fixed point $\mathrm{FP}_{2}$ arises with $\left(\alpha_{g}^{*}, \alpha_{y}^{*}, \alpha_{u}^{*}, \alpha_{u}^{*}+\alpha_{v}^{*}\right) \approx(0.91,0.42,0.40,0.13) \epsilon$ and a stable quantum vacuum $[17,38]$

$$
\alpha_{u}^{*} \geq 0, \quad \alpha_{u}^{*}+\alpha_{v}^{*} \geq 0 .
$$

A secondary fixed point in the scalar sector does not lead to a stable vacuum and has been discarded. The universal

TABLE II. Interacting fixed points and scaling exponents to leading order in $\epsilon$, with $a_{\mathrm{UV}}=\frac{2}{19}\left[(20+6 \sqrt{23})^{1 / 2}-2 \sqrt{23}\right]$ and $a_{\mathrm{IR}}=\frac{4}{3} \sqrt{3}-\left(1+\frac{4}{3} \sqrt{3}\right)^{1 / 2}$. The fixed points $\mathrm{FP}_{2}\left(\mathrm{FP}_{1,3}\right)$ are UV (IR) and physical for small positive (negative) $\epsilon$, respectively.

\begin{tabular}{cccccccccr}
\hline \hline & $\alpha_{g}^{*}$ & $\alpha_{y}^{*}$ & $\alpha_{u}^{*}$ & $\alpha_{v}^{*}$ & $\vartheta_{1}$ & $\vartheta_{2}$ & $\vartheta_{3}$ & $\vartheta_{4}$ & Type \\
\hline $\mathbf{F P}_{\mathbf{1}}$ & $-\frac{8}{75} \epsilon$ & 0 & 0 & 0 & $\frac{16}{225} \epsilon^{2}$ & $\frac{8}{25} \epsilon$ & 0 & 0 & IR \\
$\mathbf{F P}_{\mathbf{2}}$ & $\frac{52}{57} \epsilon$ & $\frac{8}{19} \epsilon$ & $\frac{2(\sqrt{23-1})}{19} \epsilon$ & $\alpha_{\mathrm{UV}} \epsilon$ & $-\frac{104}{171} \epsilon^{2}$ & $\frac{52}{19} \epsilon$ & $\frac{8}{19} \sqrt{20+6 \sqrt{23} \epsilon}$ & $\frac{16}{19} \sqrt{23} \epsilon$ & $\mathbf{U V}$ \\
$\mathbf{F P}_{\mathbf{3}}$ & $-\frac{10}{3} \epsilon$ & $-\frac{4}{3} \epsilon$ & $\frac{1-2 \sqrt{3}}{3} \epsilon$ & $\alpha_{\mathrm{IR}} \epsilon$ & $\frac{20}{9} \epsilon^{2}$ & $-10 \epsilon$ & $-8\left(1+\frac{4}{3} \sqrt{3}\right)^{1 / 2} \epsilon$ & $-\frac{32}{\sqrt{3}} \epsilon$ & $\mathbf{I R}$ \\
\hline \hline
\end{tabular}




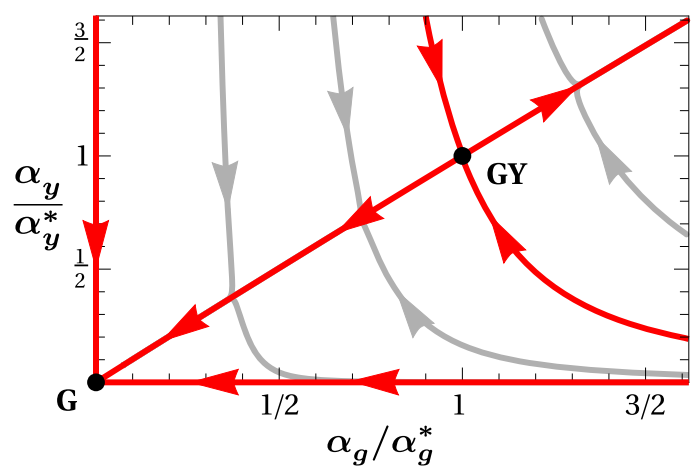

FIG. 1. (Color online) Phase diagram with asymptotic safety $(\epsilon=0.01)$, projected onto the $\left(\alpha_{g}, \alpha_{y}\right)$ plane. Arrows point from the UV to the IR. Asymptotically safe trajectories emanate from the gauge-Yukawa fixed point $\mathrm{FP}_{2}$ and run along a separatrix towards either a weakly or a strongly coupled IR regime.

exponents $\vartheta_{1}<0<\vartheta_{2,3,4}$ establish that the fixed point is UV and the scaling power law rather than logarithmic, and that the UV critical surface is one dimensional, corresponding to a single relevant coupling.

The phase diagram with RG trajectories in the $\left(\alpha_{g}, \alpha_{y}\right)$ plane is displayed in Fig. 1. Switching on mass terms for the vectorlike fermions or the scalars adds additional relevant directions (not shown), because perturbatively small anomalous dimensions cannot turn these into irrelevant operators. By the same token, higher dimensional interactions remain strictly irrelevant [22].

The separatrix that connects the UV fixed point with the free IR fixed point is shown in Fig. 2. The scale $\mu_{c}=$ $\Lambda \exp t_{c}$ with $\Lambda$ being the high scale and $\alpha_{g}\left(t_{c}\right)=\frac{2}{3} \alpha_{g}^{*}$ [17] characterizes the crossover between the two fixed points and is the analogue of $\Lambda_{\mathrm{QCD}}$ in QCD. A second separatrix exists towards a regime with strong coupling and confinement in the IR (not shown).

Finally, we note that all previously known quantum field theories in four dimensions with exact asymptotic safety involve unitary gauge symmetry and Dirac fermions [16,23-25]. In this light, the theory (3) with (6) offers the

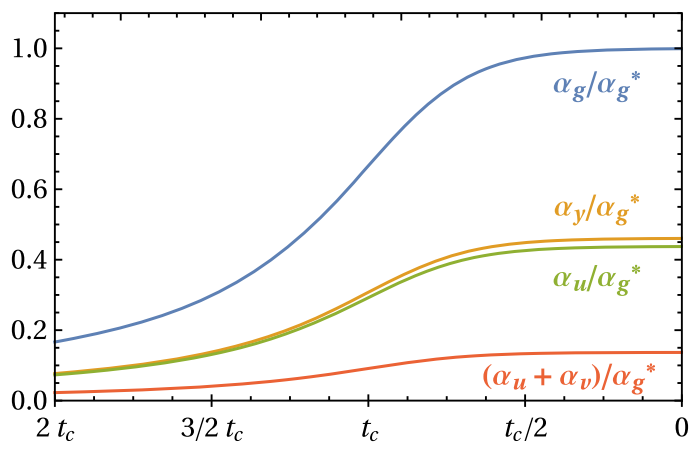

FIG. 2. (Color online) Crossover of running couplings from asymptotic safety to infrared freedom in units of $\alpha_{g}^{*}$ with $\mu_{c}=\Lambda \exp t_{c}$. first proof of existence for asymptotic safety in gauge theories with $S O(N)$ gauge symmetry, and in theories with Majorana fermions.

Symplectic gauge symmetry $S p(N)$. - Next, we turn to a theory of $N_{f}$ Majorana fermions in the fundamental representation of an $S p(N)$ gauge theory interacting with gauge-singlet complex scalar fields $H$. In our conventions $N$ is an even integer, and $S p(2) \simeq S O(3) \simeq S U(2)$. The theory has a global $S U\left(N_{f}\right)$ flavor symmetry with Weyl components transforming in the fundamental and $H$ in the two-index antisymmetric representation $H_{i j}=H_{[i j]}$. To avoid a Witten anomaly [39] $N_{f}$ has to be an even integer as well. The perturbatively renormalizable Lagrangian of the theory takes the form

$$
\begin{aligned}
L= & -\frac{1}{4} F_{\mu \nu}^{a} F^{a \mu \nu} \\
& +\operatorname{Tr}\left(\psi^{\dagger} i \sigma^{\mu} D_{\mu} \psi\right)+\operatorname{Tr}\left(\partial_{\mu} H^{\dagger} \partial^{\mu} H\right) \\
& -\frac{1}{2} y \operatorname{Tr}\left(\psi^{\top} f H \varepsilon \psi+\psi^{\dagger} f H^{\dagger} \varepsilon \psi^{*}\right) \\
& -u \operatorname{Tr}\left(H^{\dagger} H\right)^{2}-v\left(\operatorname{Tr} H^{\dagger} H\right)^{2},
\end{aligned}
$$

where we recall that gauge contractions of fermion bilinears $(\chi \xi)=\chi_{\alpha} f^{\alpha \beta} \xi_{\beta}$ are antisymmetric with $f^{\alpha \beta}=-f^{\beta \alpha}$ and $f^{\alpha \beta} f_{\beta \gamma}=-\delta^{\alpha}{ }_{\gamma}$.

A Veneziano limit is established using (4) and rescaled couplings (5). Introducing the parameter $\epsilon$ as in (4), we find the RG beta functions for all couplings to the leading nontrivial order in perturbation theory. Denoting the 't Hooft couplings (5) for the theories (3) and (8) as $\alpha_{i}^{S O}$ and $\alpha_{i}^{S p}$, respectively, we find the remarkable result that the RG beta functions (6) for the theory (3) agree exactly with those of the theory (8), after the identification of couplings

$$
\alpha_{i}^{S O}=\alpha_{i}^{S p} .
$$

Consequently the fixed points and scaling exponents $\left(\mathrm{FP}_{1}\right.$ and $\mathrm{FP}_{2}$ in Table II), and the RG trajectories and phase diagrams (Figs. 1 and 2) of the theories (3) and (8) are identical in the Veneziano limit. However, we also note that the equivalence is mildly violated beyond the Veneziano limit at large yet finite $N$ and $N_{f}$ due to subleading corrections of order $1 / N$ and $1 / N_{f}$, which arise with the same magnitude but opposite sign.

Finally, we emphasize that the theory (8) yields the first rigorous example for asymptotic safety in a symplectic gauge theory coupled to matter. The result thus establishes that asymptotic safety can be achieved in $4 \mathrm{D}$ quantum field theories with any of the nonexceptional gauge groups, and for sufficiently large $N$.

\section{DIRAC FERMIONS}

Next, we consider theories of $N_{f}$ Dirac fermions $\Psi_{i}$ interacting with non-Abelian gauge fields and gauge-singlet 
complex scalar fields $H_{i j}$. The theories have a global $S U\left(N_{f}\right) \times S U\left(N_{f}\right)$ flavor symmetry with the elementary scalars $H$ transforming in the bifundamental. The perturbatively renormalizable Lagrangian is given by

$$
\begin{aligned}
L= & -\frac{1}{4} F_{\mu \nu}^{a} F_{a}^{\mu \nu}+\operatorname{Tr}(\bar{\Psi} i \not \supset \Psi)+\operatorname{Tr}\left(\partial_{\mu} H^{\dagger} \partial^{\mu} H\right) \\
& -y \operatorname{Tr}\left(\bar{\Psi}_{L} H \Psi_{R}+\bar{\Psi}_{R} H^{\dagger} \Psi_{L}\right)-u \operatorname{Tr}\left(H^{\dagger} H\right)^{2} \\
& -v\left(\operatorname{Tr} H^{\dagger} H\right)^{2},
\end{aligned}
$$

where $F_{\mu \nu}^{a}$ denotes the non-Abelian field strength, the trace sums over all indices and the decomposition $\Psi=\Psi_{L}+\Psi_{R}$ with $\Psi_{L / R}=\frac{1}{2}\left(1 \pm \gamma_{5}\right) \Psi$ is understood. Because of the fermions being vectorlike, gauge anomalies cancel by design, and no restriction on their representations apply. In addition to the gauge coupling $g$ and the Yukawa coupling $y$, we observe two independent quartic self-interactions $u$ and $v$, which provides us with a set of four canonically marginal couplings $\{g, y, u, v\}$. In the following, we consider the Dirac fermions in the fundamental gauge representation of $S U(N), S O(2 N)$, and $S p(2 N)$. We also establish a Veneziano limit using parameter (4) and adopt the same set of "t Hooft couplings (5) as in the cases with Majorana fermions.

Unitary gauge symmetry $S U(N)$.- - For unitary gauge groups, theory (10) has been studied in a number of works $[16,17,23,40,41]$. In the regime with asymptotic freedom, it can display a Banks-Zaks fixed point. Once asymptotic freedom is lost, it develops a weakly interacting asymptotically safe UV fixed point [16] with a stable quantum vacuum [17]. The corresponding UV conformal window has been determined up to the complete next-to-next-toleading order in perturbation theory, which is three loop in the gauge and two loop in the Yukawa and quartic couplings [23].

The main observation here is that theory (10) with $S U$ gauge symmetry and Dirac fermions is intimately related to theories (3) with $S O$ and to (8) with $S p$ gauge symmetry and Majoranas. Introducing the parameter $\epsilon$ as in (4) and denoting the couplings (5) for the theory (10) with unitary gauge symmetry as $\alpha_{i}^{S U}$, we find that beta functions in the Veneziano limit are identical to those of the theories (3) and (8), given by (6), provided we rescale the 't Hooft couplings by a factor of 2 ,

$$
\alpha_{i}^{S O}=\alpha_{i}^{S p}=2 \alpha_{i}^{S U}
$$

Consequently fixed points are either of the Banks-Zaks $\left(\mathrm{FP}_{1}\right)$ or the gauge-Yukawa type $\left(\mathrm{FP}_{2}\right)$ and take the values given in Table II after rescaling. Phase diagrams and RG trajectories in these theories are also identical up to (11), and given by Figs. 1 and 2. Most notably, universal scaling exponents, which are insensitive to the normalization of couplings, are identical between the two theories, and take the values given in Table II.

$S O(2 N)$ gauge symmetry.-Next, we consider settings with $N_{f}$ Dirac fermions in the fundamental representation of $S O(2 N)$ gauge symmetry, again coupled to scalars in the bifundamental two-index representation of the global $S U\left(N_{f}\right) \times S U\left(N_{f}\right)$ flavor symmetry, and with action (10). Notice that since Dirac fermions have twice as many degrees of freedom (d.o.f.) as Majorana fermions, and to ensure that the definition for the small parameter $\epsilon$ (4) remains unchanged, the dimension of the gauge group has been taken twice as large as in the case with $S U$ gauge symmetry. Then, to the leading order in perturbation theory and in the Veneziano limit, we find

$$
\begin{aligned}
& \beta_{g}=\alpha_{g}^{2}\left[\frac{2}{3} \epsilon+\left(\frac{25}{4}+\frac{13}{6} \epsilon\right) \alpha_{g}-\frac{1}{2}\left(\frac{11}{2}+\epsilon\right)^{2} \alpha_{y}\right], \\
& \beta_{y}=\alpha_{y}\left[\left(\frac{15}{2}+\epsilon\right) \alpha_{y}-3 \alpha_{g}\right], \\
& \beta_{u}=8 \alpha_{u}^{2}+4 \alpha_{y} \alpha_{u}-\left(\frac{11}{2}+\epsilon\right) \alpha_{y}^{2}, \\
& \beta_{v}=4 \alpha_{v}^{2}+16 \alpha_{u} \alpha_{v}+12 \alpha_{u}^{2}+4 \alpha_{y} \alpha_{v}
\end{aligned}
$$

for models (10) with orthogonal gauge symmetry.

In stark contrast to the previous examples, no interacting UV fixed points are found as soon as asymptotic freedom is absent [18]. However, the beta functions (12) admit interacting fixed points provided the theory is asymptotically free $(\epsilon<0)$. These are either of the Banks-Zaks $\left(\mathrm{FP}_{1}\right)$ or of the gauge-Yukawa-type $\left(\mathrm{FP}_{3}\right)$, with fixed point coordinates and scaling exponents summarized in Table II. The gauge-Yukawa fixed point $\mathrm{FP}_{3}$ at $\left(\alpha_{g}^{*}, \alpha_{y}^{*}, \alpha_{u}^{*}\right.$, $\left.\alpha_{u}^{*}+\alpha_{v}^{*}\right) \approx-(3.33,1.33,1.23,0.58) \epsilon$ also displays a stable quantum vacuum (7).

The universal exponents $0<\vartheta_{1,2,3,4}$ establish that the fixed point $\mathrm{FP}_{3}$ is fully attractive in all canonically marginal couplings, thus corresponding to an IR sink [20], and that the scaling is power law rather than logarithmic. The phase diagram in regimes with asymptotic freedom is shown in Fig. 3. We notice that the Banks-Zaks fixed point is parametrically small compared to the gauge-Yukawa fixed point (see the inset in Fig. 3). It implies that the UV critical surface at the Gaussian fixed point, which is two dimensional, effectively becomes one dimensional, given by the separatrix connecting the Gaussian and the GY fixed point.

$S p(2 N)$ gauge symmetry.-Finally, we turn to the models (10) with $N_{f}$ Dirac fermions in the fundamental of $S p(2 N)$ gauge symmetry, coupled to scalars in the bifundamental two-index representation of the global $S U\left(N_{f}\right) \times S U\left(N_{f}\right)$ flavor symmetry. Using (4), introducing couplings as in (5), and following the same steps as before, we find once more that the beta functions in the Veneziano limit come out identical to those found in (12) 


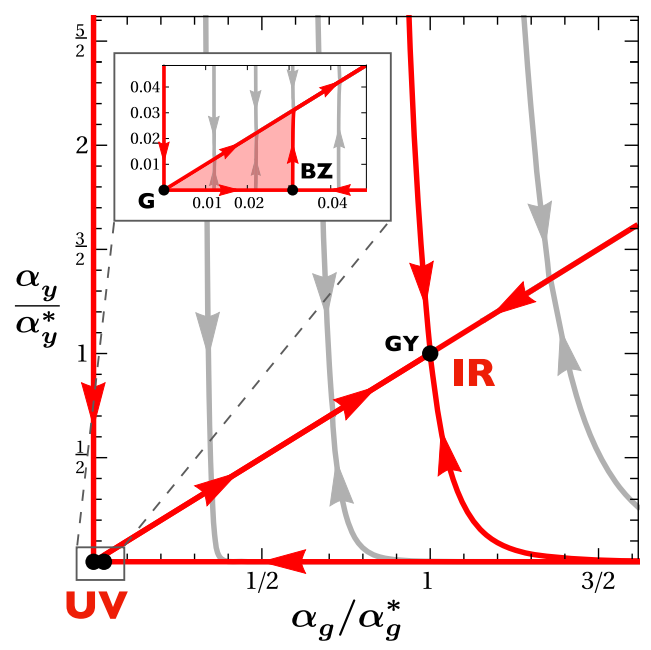

FIG. 3. (Color online) Phase diagram with asymptotic freedom $(\epsilon=-0.01)$ projected onto the $\left(\alpha_{g}, \alpha_{y}\right)$ plane. Arrows point from the UV to the IR. Dots show the Gaussian (G), the Banks-Zaks (BZ), and the infrared gauge-Yukawa (GY) fixed points. The inset highlights the two dimensionality of the UV critical surface (red shaded area), which becomes effectively one dimensional in the crossover to the IR.

after a straight identification of couplings (9). It follows that the running of couplings, the phase diagrams, and the conformal critical points of theories (10) are identical, irrespective of whether we impose an orthogonal or symplectic gauge symmetry.

\section{LARGE $N$ EQUIVALENCES}

In this section, we investigate the kinematical equivalences detected in the previous sections from the point of view of weak-coupling dualities and orbifold projections, and discuss implications for asymptotic safety.

Negative dimensionality theorems. - Some of our results can be understood with the help of so-called negative dimensionality theorems [42-46]. They state that for any $S O(L)$ invariant scalar there exists a corresponding $S p(L)$ invariant scalar, and vice versa, obtained by exchanging symmetrizations and antisymmetrizations, replacing the $S O(L)$ symmetric bilinear invariant $h_{\alpha \beta}$ by the $S p(L)$ antisymmetric bilinear invariant $f_{\alpha \beta}$, and replacing $L$ by $-L$. Similarly, for any $S U(M)$ invariant scalar exchanging symmetrizations and antisymmetrizations is equivalent to replacing $M$ by $-M$. Schematically, we write the theorems as

$$
S O(L)=\overline{S p}(-L), \quad S U(M)=\overline{S U}(-M),
$$

where overlines indicate the transposition of Young tableaux for all representations, corresponding precisely to the interchange of symmetrization and antisymmetrization [42-46].
Symplectic vs orthogonal gauge groups.-Let us now clarify how the negative dimensionality theorems impact our models. On the level of the local symmetries in the models with Majorana fermions (3) and (8), the relations (13) interchange orthogonal and symplectic gauge theories. When applied to the global $S U\left(N_{f}\right)$ symmetry the transposition of global representations accounts for the different symmetrizations of the scalars, interchanging $H_{(i j)}$ with $H_{[i j]}$, and all of this accompanied by the analytic continuation of field multiplicities towards negative values

$$
N \mapsto-N, \quad N_{f} \mapsto-N_{f} .
$$

Fingerprints of the negative dimensionality theorems (13) can be seen on the level of the renormalization group equations. For theories with Majorana fermions, we have confirmed at the leading orders in perturbation theory that the beta functions for the gauge, Yukawa, and quartic couplings of the theory (3) are identical to the beta functions of the theory (8) for any $N$ and $N_{f}$, provided we make the replacement (14) in the latter together with $\left\{g^{2}, y^{2}, u, v\right\} \mapsto\left\{-g^{2},-y^{2},-u, v\right\}$. This implies that the gauge, Yukawa, and single and double trace quartic 't Hooft couplings

$$
\left\{N g^{2}, N y^{2}, N_{f} u, N_{f}^{2} v\right\}
$$

are strictly invariant and remain positive even within the theory that has negative $N$ and $N_{f}$, as they must [47]. For pure quantum gauge theories the invariance of $N g^{2}$ under (13) is explained in [43]. Moreover, we have also confirmed that the exact same equivalence holds true for beta functions and running couplings in theories with Dirac fermions (10) coupled to orthogonal or symplectic gauge fields. In summary, we conclude that the negative dimensionality theorems manifest themselves in the quantum theory through the equivalence of ' $t$ Hooft couplings and their beta functions for any $N$ and any $N_{f}$. We expect this equivalence to hold true to any order in the perturbative loop expansion.

For positive $N$ and $N_{f}$, the large $N$ equivalence of the theories (3) and (8), and of the theories (10) with either $S O$ or $S p$ gauge symmetry, is now simple to understand, the key point being that the explicit dependence of beta functions on field multiplicities arises, in the Veneziano limit, only through the parameter $\epsilon$ given in (4). Since $\epsilon$ is insensitive to the combined sign change (14), the mapping of negative field multiplicities in the partner theory back to positive ones leaves all beta functions for ' $t$ Hooft couplings (15) invariant. The price to pay (for having positive field multiplicities on either side of the duality) is that the equivalence holds only in the large $N$ limit. In fact, in either theory the subleading corrections start at order $1 / N$ and $1 / N_{f}$ and enter with the same magnitude but opposite signs (once more owing to the negative dimensionality theorems) 


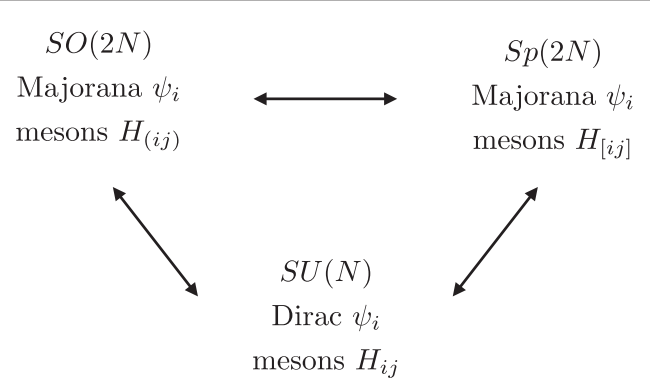

FIG. 4. Triality of asymptotic safety, and large $N$ equivalences among matter-gauge theories with global $S U\left(2 N_{f}\right)$ flavor symmetry and Majorana fermions (top), and a theory with a global $S U\left(N_{f}\right) \times S U\left(N_{f}\right)$ flavor symmetry and Dirac fermions (bottom). The horizontal arrow emphasizes that RG flows, phase diagrams, and critical points are identical. Top-down arrows indicate equivalence after orbifold reduction.

thus breaking the duality beyond large $N$. This pattern explains the equivalence of beta functions for ' $t$ Hooft couplings as well as the structure of subleading corrections found in the previous sections, and illustrated in Figs. 4 and 5.

There are two further points worth noting with regards to large $N$ equivalences. First, counting the number of gauge fields, Weyl fermions, and real scalar fields in either of these, we find that dual theories have the exact same number of d.o.f. (see model 1 vs model 2, and model 4 vs model 5 in Table III). This no longer holds true beyond large $N$. Second, we also emphasize that dual theories described here, in all cases, have the same global symmetry but different gauge symmetry. This supports the view that global symmetry is a property of the system, whereas gauge symmetry is a property of the description of the system [48].

Unitary gauge groups.-For the theories with Dirac fermions (10) and $S U$ gauge symmetry we confirm that beta functions for ' $t$ Hooft couplings are mapped onto themselves under the replacement (13) and (14), valid for all $N$. Moreover, subleading corrections in the large $N$ limit arise as inverse even powers of field multiplicities $1 / N^{2}$ and $1 / N_{f}^{2}$ and are insensitive to a change in sign (14), meaning that the theory is effectively self-dual and mapped onto itself for any $N, N_{f}$, as it must. The result generalizes to $S U$ gauge theories with matter sectors different from (10).

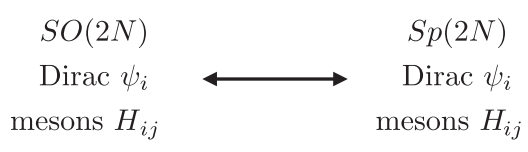

FIG. 5. Duality of conformal fixed points, and large $N$ equivalences of matter-gauge theories with global $S U\left(N_{f}\right) \times$ $S U\left(N_{f}\right)$ flavor symmetry.

Orbifold equivalence.-We now turn to the equivalence of theories between Dirac fermions coupled to unitary gauge fields, and Majorana fermions coupled to orthogonal or symplectic gauge fields, illustrated in Fig. 4. Theories have different global symmetries, and those with Dirac fermions contain exactly half as many gauge, Weyl, and scalar d.o.f. as those with Majorana fermions in the Veneziano limit (see models 1 and 2 vs model 3 in Table III). Still, after the identification of couplings via the map (11), all three theories have identical beta functions, phase diagrams, conformal fixed points, and scaling exponents. This pattern suggests that the theories are related by orbifolding.

Orbifold projections in quantum field theory link a parent theory to a child theory with the help of a discrete subgroup of the parent's global symmetry [5-10,12,13] (see $[11,14]$ for reviews). Orbifolding eliminates those d.o.f. from the parent theory that are not invariant under the discrete subgroup, leading to the child theory. At the perturbative level, orbifold equivalence is based on the observation that planar diagrams of the parent and child theories coincide to all loop orders, possibly up to a rescaling of couplings, and that correlation functions of gauge-invariant operators obey the same set of closed equations [5]. It has also been shown that the equivalence of theories holds nonperturbatively as long as the global symmetry used for the orbifolding is not broken spontaneously [12].

In the setting illustrated in Fig. 4, the Majorana models (3) and (8) with $S O(2 N)$ or $S p(2 N)$ gauge symmetry and $S U\left(2 N_{f}\right)$ global symmetry represent parent theories. Then, using a suitable $\mathbb{Z}_{2}$ symmetry in the gauge and flavor groups [13] leads in both cases to the child theory (10) with Dirac fermions, $S U(N)$ gauge symmetry, and $S U\left(N_{f}\right) \times$ $S U\left(N_{f}\right)$ global symmetry [49]. In the Veneziano limit, the

TABLE III. Gauge, fermionic, and scalar d.o.f. in the Veneziano limit of models discussed in the main text.

\begin{tabular}{lcccccccc}
\hline \hline Model & Lagrangian & $\begin{array}{c}\text { Gauge } \\
\text { symmetry }\end{array}$ & $\begin{array}{c}\text { Global } \\
\text { symmetry }\end{array}$ & $\begin{array}{c}\text { Gauge } \\
\text { bosons }\end{array}$ & $\begin{array}{c}\text { Fermion } \\
\text { type }\end{array}$ & $\begin{array}{c}\text { Weyl } \\
\text { components }\end{array}$ & Scalars & $\begin{array}{c}\text { Real scalar } \\
\text { components }\end{array}$ \\
\hline 1 & Eq. (3) & $S O(2 N)$ & $S U\left(2 N_{f}\right)$ & $2 N^{2}$ & Majorana & $2 N \cdot 2 N_{f}$ & $H_{(i j)}$ & $4 N_{f}^{2}$ \\
2 & Eq. (8) & $S p(2 N)$ & $S U\left(2 N_{f}\right)$ & $2 N^{2}$ & Majorana & $2 N \cdot 2 N_{f}$ & $H_{[i j]}$ & $4 N_{f}^{2}$ \\
3 & Eq. (10) & $S U(N)$ & $S U\left(N_{f}\right) \times S U\left(N_{f}\right)$ & $N^{2}$ & Dirac & $N \cdot 2 N_{f}$ & $H_{i j}$ & $2 N_{f}^{2}$ \\
4 & Eq. (10) & $S O(2 N)$ & $S U\left(N_{f}\right) \times S U\left(N_{f}\right)$ & $2 N^{2}$ & Dirac & $2 N \cdot 2 N_{f}$ & $H_{i j}$ & $2 N_{f}^{2}$ \\
5 & Eq. (10) & $S p(2 N)$ & $S U\left(N_{f}\right) \times S U\left(N_{f}\right)$ & $2 N^{2}$ & Dirac & $2 N \cdot 2 N_{f}$ & $H_{i j}$ & $2 N_{f}^{2}$ \\
\hline \hline
\end{tabular}


orbifold equivalence between parent and child theories is exact, explaining the links observed in Fig. 4. The factor of 2 that appears in the rescaling (11) reflects that the parent theories contain twice as many gauge, Weyl, and scalar d.o.f. as the child theory; see Table III.

In the literature, some orbifold/orientifold reductions have been reported that relate supersymmetric with nonsupersymmetric theories $[9,11]$. On the account that asymptotic safety in supersymmetry necessitates the gauge group to be semisimple [25,50], however, we do not expect to find a supersymmetric parent for the nonsupersymmetric theories with weakly coupled ultraviolet fixed points and simple gauge group studied here.

Dirac vs Majorana fermions.-Another important observation of this study is that $S O$ and $S p$ gauge theories with Majorana fermions and elementary mesons can develop asymptotically safe UV fixed points while their counterparts with Dirac fermions cannot. To appreciate the origin for this we write the leading loop contributions to the gauge and Yukawa beta functions as $\partial_{t} \alpha_{g}=\alpha_{g}^{2}(-B+$ $\left.C \alpha_{g}-D \alpha_{y}\right)$ and $\partial_{t} \alpha_{y}=\alpha_{y}\left(E \alpha_{y}-F \alpha_{g}\right)$ with $C, D$ and $B, E, F$ denoting universal two-loop and one-loop coefficients, respectively. A necessary condition for weakly interacting UV fixed points is given by [18]

$$
C^{\prime} \equiv C-D F / E<0
$$

A generic asymptotically nonfree theory $(B<0)$ has loop coefficients $C, D, E, F>0$ [18], implying $C^{\prime} \leq C$. The condition (16) states that asymptotic safety requires the (Yukawa-shifted) two-loop term to become negative, $C^{\prime}<0$. In all theories with exact asymptotic safety (meaning $\mathrm{FP}_{2}$ in Table II) we find the universal shift

$$
C^{\prime} / C=-\frac{38}{325},
$$

assuming small $0<\epsilon \ll 1$. Hence, the Yukawa interactions roughly induce a $-112 \%$ correction to the two-loop gauge coefficient, which is large enough to change the sign of $C$ and to enable asymptotic safety.

Replacing Majorana by Dirac fermions in the theories with $S O$ or $S p$ gauge symmetry effectively changes the scalar matter content. In fact, adjusting $N$ and $N_{f}$ such that theories display the same number of gauge fields and Weyl fermions, we find that the settings with Dirac fermions only feature half as many scalar d.o.f. (see models 1 and 2 vs models 4 and 5 in Table III). Although scalars are gauge singlets, they propagate in loops and modify the Yukawa loop coefficient $E$, which is proportional to the number of d.o.f. [51]. Here, the coefficient $\left.E\right|_{\text {Majorana }} \sim 2 N_{f}+2 N$ reduces down to $\left.E\right|_{\text {Dirac }} \sim N_{f}+2 N$ and gives the first term in $\beta_{y}$ of (6) and (12), respectively, after 't Hooft normalization. With all other coefficients untouched, we find

$$
C^{\prime} / C=\frac{4}{125}
$$

instead of (17). This corresponds to a $-97 \%$ correction of the two-loop gauge coefficient $C$, which is narrowly too small to change the overall sign of $C$. The result (18) explains why models with Dirac fermions and $S O$ or $S p$ gauge symmetry may display interacting infrared fixed points, but cannot develop interacting ultraviolet ones, much unlike their counterparts with Majoranas.

As a final remark, we note that the ratio (18) also dictates the ratio of gauge couplings at the Banks-Zaks fixed point compared to the gauge-Yukawa fixed point in asymptotically free $S O$ and $S p$ gauge theories with Dirac fermions (10). There, we found that $\alpha_{g}^{\mathrm{BZ}} /\left.\alpha_{g}^{\mathrm{GY}}\right|_{*}=C^{\prime} / C$ provided that $0<-\epsilon \ll 1$ (see $\mathrm{FP}_{1}$ and $\mathrm{FP}_{3}$ in Table II). Hence, the parametric smallness of the ratio of fixed point couplings, as observed in Fig. 3, can now be attributed to the "near miss" of asymptotic safety due to (18).

\section{DISCUSSION AND CONCLUSIONS}

As a proof of principle, we have established that asymptotic safety arises in matter-gauge theories with Majorana fermions, and in theories with $S O$ and $S p$ gauge symmetry (3) and (8). Together with the earlier discovery of asymptotic safety with Dirac fermions in $S U$ gauge theories (10), our results clarify that interacting ultraviolet fixed points can readily be realized for either type of fermions and for any of the classical gauge groups. Intriguingly, though, $S U$ gauge symmetry does require fermions to be Dirac, whereas $S O, S p$ gauge symmetry does require fermions to be Majorana.

We have also put forward new classes of large $N$ equivalences between seemingly different gauge-matter theories. Equivalences between pure $S U, S O$, and $S p$ gauge theories in the planar limit have been known for a long time. Here, we have explained how equivalences arise among theories with different local symmetries, different matter content, and, possibly, different global symmetries. Invariably, they imply identical all-order RG flows, phase diagrams, and conformal critical points (Table II). Examples (Table III) include a triality of asymptotic safety (Figs. 1 and 4) or dualities among theories with identical infrared critical points (Figs. 3 and 5). Based on the underlying structure, many more large $N$ equivalences arise in gauge theories with matter and Yukawa interactions, also offering new directions for orbifold reductions [11,49].

Finally, we note that our theories, at interacting fixed points, correspond to unitary conformal field theories. This link allows the extraction of conformal data such as scaling dimensions [16,23-25] or structure coefficients [52] directly from the renormalization group [53], and in a manner complementary to the conformal bootstrap [54]. It will be interesting to see whether the equivalences discovered here (Figs. 4 and 5) extend to all conformal data. This is left for future work. 
[1] C. Lovelace, Nucl. Phys. B201, 333 (1982).

[2] N. Seiberg, Nucl. Phys. B435, 129 (1995).

[3] J. M. Maldacena, Int. J. Theor. Phys. 38, 1113 (1999); Adv. Theor. Math. Phys. 2, 231 (1998).

[4] S. Kachru and E. Silverstein, Phys. Rev. Lett. 80, 4855 (1998).

[5] M. Bershadsky and A. Johansen, Nucl. Phys. B536, 141 (1998).

[6] M. Schmaltz, Phys. Rev. D 59, 105018 (1999).

[7] J. Erlich and A. Naqvi, J. High Energy Phys. 12 (2002) 047.

[8] M. J. Strassler, arXiv:hep-th/0104032.

[9] A. Armoni, M. Shifman, and G. Veneziano, Nucl. Phys. B667, 170 (2003).

[10] P. Kovtun, M. Unsal, and L. G. Yaffe, J. High Energy Phys. 07 (2005) 008.

[11] G. V. Dunne and M. Ünsal, Annu. Rev. Nucl. Part. Sci. 66, 245 (2016).

[12] P. Kovtun, M. Unsal, and L. G. Yaffe, J. High Energy Phys. 06 (2007) 019.

[13] M. Hanada and N. Yamamoto, J. High Energy Phys. 02 (2012) 138.

[14] B. Lucini and M. Panero, Phys. Rep. 526, 93 (2013).

[15] D. Bailin and A. Love, Nucl. Phys. B75, 159 (1974).

[16] D. F. Litim and F. Sannino, J. High Energy Phys. 12 (2014) 178.

[17] D. F. Litim, M. Mojaza, and F. Sannino, J. High Energy Phys. 01 (2016) 081.

[18] A. D. Bond and D. F. Litim, Eur. Phys. J. C 77, 429 (2017).

[19] A. Codello, K. Langæble, D. F. Litim, and F. Sannino, J. High Energy Phys. 07 (2016) 118.

[20] A. D. Bond, G. Hiller, K. Kowalska, and D. F. Litim, J. High Energy Phys. 08 (2017) 004.

[21] A. Bond and D. F. Litim, Proc. Sci., LATTICE2016 (2017) 208.

[22] T. Buyukbese and D. F. Litim, Proc. Sci., LATTICE2016 (2017) 233.

[23] A. D. Bond, D. F. Litim, G. Medina Vazquez, and T. Steudtner, Phys. Rev. D 97, 036019 (2018).

[24] A. D. Bond and D. F. Litim, Phys. Rev. D 97, 085008 (2018).

[25] A. D. Bond and D. F. Litim, Phys. Rev. Lett. 119, 211601 (2017).

[26] K. Kowalska, A. Bond, G. Hiller, and D. F. Litim, Proc. Sci., EPS-HEP2017 (2017) 542.
[27] A. D. Bond and D. F. Litim, Phys. Rev. Lett. 122, 211601 (2019).

[28] D. Barducci, M. Fabbrichesi, C. M. Nieto, R. Percacci, and V. Skrinjar, J. High Energy Phys. 11 (2018) 057.

[29] G. Hiller, C. Hormigos-Feliu, D. F. Litim, and T. Steudtner, arXiv:1905.11020.

[30] G. Hiller, C. Hormigos-Feliu, D. F. Litim, and T. Steudtner, arXiv:1910.14062.

[31] G. Veneziano, Nucl. Phys. B159, 213 (1979).

[32] M. E. Machacek and M. T. Vaughn, Nucl. Phys. B222, 83 (1983).

[33] M. E. Machacek and M. T. Vaughn, Nucl. Phys. B236, 221 (1984).

[34] M. E. Machacek and M. T. Vaughn, Nucl. Phys. B249, 70 (1985).

[35] M.-x. Luo, H.-w. Wang, and Y. Xiao, Phys. Rev. D 67, 065019 (2003).

[36] G. 't Hooft, Nucl. Phys. B72, 461 (1974).

[37] M. A. Luty, J. Polchinski, and R. Rattazzi, J. High Energy Phys. 01 (2013) 152.

[38] E. Gildener, Phys. Rev. D 13, 1025 (1976).

[39] E. Witten, Phys. Lett. 117B, 324 (1982).

[40] H. Terao and A. Tsuchiya, arXiv:0704.3659.

[41] D. B. Kaplan, J.-W. Lee, D. T. Son, and M. A. Stephanov, Phys. Rev. D 80, 125005 (2009).

[42] R. C. King, J. Math. Phys. (N.Y.) 12, 1588 (1971).

[43] R. L. Mkrtchian, Phys. Lett. 105B, 174 (1981).

[44] P. Cvitanovic and A. D. Kennedy, Phys. Scr. 26, 5 (1982).

[45] P. Cvitanovic, Group Theory: Birdtracks, Lie's and Exceptional Groups (Princeton University Press, Princeton, 2008).

[46] R. L. Mkrtchyan and A. P. Veselov, J. Math. Phys. (N. Y.) 52, 083514 (2011).

[47] F. J. Dyson, Phys. Rev. 85, 631 (1952).

[48] E. Witten, Nat. Phys. 14, 116 (2018).

[49] D. F. Litim and T. Steudtner (to be published).

[50] S. P. Martin and J. D. Wells, Phys. Rev. D 64, 036010 (2001).

[51] A. D. Bond, D. F. Litim, and T. Steudtner (to be published).

[52] A. Codello, M. Safari, G. P. Vacca, and O. Zanusso, Eur. Phys. J. C 78, 30 (2018).

[53] J. Cardy, in Scaling and Renormalization in Statistical Physics, Cambridge Lecture Notes in Physics (Cambridge University Press, Cambridge, England, 1996).

[54] D. Poland, S. Rychkov, and A. Vichi, Rev. Mod. Phys. 91, 015002 (2019). 